\title{
A novel approach to evaluating the reproducibility of a replication technique for the manufacture of electroconductive replicas for use in quantitative clinical dental wear studies.
}

\author{
R.G. Chadwick ${ }^{1}$ \\ H.L. Mitchell ${ }^{2}$ \\ S. Ward ${ }^{1}$ \\ ${ }^{1}$ The Dental School, University of Dundee, UK. \\ ${ }^{2}$ School of Engineering, The University of Newcastle, Australia.
}

\begin{abstract}
All correspondence concerning this paper should be addressed to Dr R G Chadwick, Unit of Restorative Dentistry, The Dental School, Park Place, Dundee, DD1 4HN, Scotland, UK.
\end{abstract}

Running Title: A novel method of evaluating the reproducibility of electroconductive replicas. 
Summary - The assessment of the progression of tooth surface loss has until recently been limited to either the application of subjective ranking scales or visual comparison of sequential study casts. The development of quantitative measuring techniques offers the potential of greater accuracy and sensitivity. As direct intra-oral measurement is problematical such approaches often utilise impressions of the teeth, recorded at different epochs, to construct replicas for mapping and comparison. This in vitro investigation sought to determine the reproducibility of such an approach taking into account the total process chain. Two inlay cavities (one large, one small) were prepared in the palatal aspect of a plastic maxillary central incisor and restored with two flush fitting inlays. A series of impressions of this tooth were recorded, using a special tray and an addition cured light bodied silicone impression material (President, Coltene, Switzerland), with (a) both inlays in (b) both inlays out (c) large inlay out \& small inlay in (d) large inlay in \& small inlay out - a total of 16 impressions. Electroconductive replicas were fabricated from these and mapped using a computer controlled probe. Each series simulated wear of the tooth. A Surface Matching and Difference Detection Algorithm was then used to compare each series of replicas and calculate the proportion of the surface undergoing simulated wear by a direct comparison of (a) matched to (b) or, indirectly as the summation of the results of matches of (a) with (c) and (a) with (d). The mean proportion of the surface with wear calculated directly was $26.6 \%(\mathrm{SD}=0.6)$ and indirectly $26.1 \%(\mathrm{SD}=0.5)$. A One Way Analysis of Variance revealed no significant difference $(\mathrm{P}>0.05)$. It is concluded that determining wear by this method is highly reproducible.

Keywords: Replicas, Reproducibility, Electroconductive, Erosion. 


\section{Introduction}

Tooth surface erosion is a condition that, according to the 1993 National Survey of Child Dental Health in the United Kingdom (O’Brian, 1994), and the more recent National Diet and Nutrition Survey (2000), affects just in excess of $50 \%$ of children. Monitoring the success or otherwise of preventative measures (such as lifestyle changes), initiated professionally by the presence of the condition, is complicated by the lack of fixed intraoral reference points from which to make measurements. A potential solution, reported previously (Chadwick et al., 1997) is to record addition cured silicone impressions of the dentition at different epochs. These facilitate fabrication of electroconductive replicas suitable for mapping, by means of a computer-controlled probe. The resultant maps, termed Digital Terrain Models (DTM’s), may be compared using a Surface Matching and Difference Detection Algorithm (SMADDA). This technique mathematically seeks regions of coincidence and conflict between DTM's of the same tooth, at different epochs, to determine the degree of surface loss that has occurred (Mitchell and Chadwick, 1998, 1999). A major factor determining the success of such an approach however, is the replication process itself. We have reported previously on this by both determining the ability of replication to reproduce accurately a known step height and reproduce a palatal

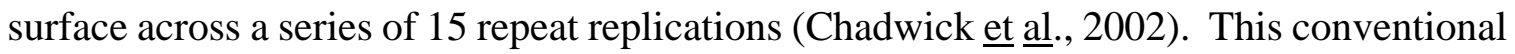
approach indicated reproduction of step height to within $3 \mu \mathrm{m}$ and a constancy of surface topography reproduction of 99.6\%. The aim of this work was to further test the replication process by a novel means, as described in this paper, and report upon the outcome.

\section{Materials and methods}


The concept of the novel procedure is as follows. A single maxillary central incisor, prepared to house two flush fitting and removable inlays on its palatal aspect (Figure 1), formed the test bed for this work. The incorporation of inlays within this tooth enabled reproducible changes in surface topography to be carried out, simulating palatal tooth surface loss, by their removal. By recording impressions of the palatal aspect of this test tooth in the following states:

1. Both inlays in situ

2. Both inlays out

3. Large inlay out and small inlay in

4. Large inlay in and small inlay out

And subsequently producing replicas it is possible to ascertain the proportion of the test tooth undergoing simulated palatal tooth surface loss. This involves fabricating electroconductive replicas from each impression, mapping their surfaces and comparing them using a SMADDA. By undertaking matches of the replica of State 1 with each of the replicas of the test tooth in States 2, 3 and 4 the apparent proportions of the palatal surface occupied by the inlays can be determined in a variety of ways. Should these agree it can be assumed that the replication procedure is reproducible.

The methods and materials of this investigation may be best detailed under the subheadings: Replica acquisition, Surface Mapping and Surface Comparison. 


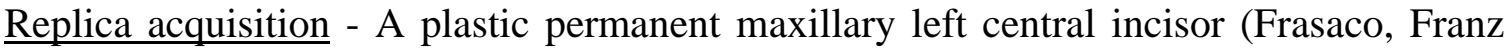
Sachs 410, Germany), intended for phantom head exercises, was mounted using a light curing special tray material (Triad, DeTrey Dentsply, Surrey, UK) upon a circular flat stainless steel disc of $53 \mathrm{~mm}$ diameter. This was arranged in such a way that, when viewed from above, the palatal surface was uppermost and clearly visible. A special tray was fabricated with $3 \mathrm{~mm}$ tray spacing, using the same custom tray material as used to mount the central incisor, to enable impressions of the palatal surface to be recorded.

1. Prior to recording any impressions two saucerised inlay cavities were prepared on the palatal aspect of this tooth - one small (occupying approximately $10 \%$ of the tooth surface) and one large (occupying approximately $16 \%$ of the tooth surface). These cavities were provided with two gold flush fitting removable inlays, conforming to the tooth's original anatomic form, using a conventional lost wax casting technique. A series of four impressions of this tooth were recorded, using the special tray, with the inlays in the states as described above. This procedure was repeated a further three times so that in total four replicas of each state were made from four separate impressions resulting in a total of 16 replicas.

A full account of the impression and replication process is given in Chadwick et al., 2002. In summary however, tray adhesive (Universal Adhesive, Bayer Dental, Germany) was applied to the fit surface of the special tray and allowed to dry. An addition cured light bodied silicone impression material (President, Coltene, Switzerland) was used to record the impression within the tray, which, upon withdrawal was inspected closely for surface imperfections. The surface of each satisfactory silicone impression was painted with two 
coats of a high silver content electroconductive paint (RS Silver Paint, RS Components, Corby, Northants, UK) and, once dry, a cyanoacrylate based gel material (Zapit®, Dental Ventures of America Inc., USA) was applied to back up the painted surface and reinforce it. In order to increase the thickness of the resultant replica and to facilitate both handling and mounting upon the mapping device, this was further backed up with die stone (Miles Dental Products, South Bend, IN, USA), mixed according to the manufacturer's recommended powder: liquid ratio, before being removed from the impression. Thus, upon removal from the impression, an electroconductive replica resulted whose surface was composed of a layer of silver paint conforming to the surface dimensions of the tooth under investigation as captured by the impression.

Surface Mapping - Each replica was transferred to the mapping device that was a development of that described by Chadwick et al. (1997). It consisted of a precision x, y table (Daedal, Pittsburgh, USA) motorised by the addition of two computer controlled stepper motors (RS Components Ltd., Corby, UK) that controlled precisely the position of the table in the horizontal $\mathrm{x}, \mathrm{y}$ planes. In addition, a third geared stepper motor under computer control, mounted perpendicular to the motorised table, governed the position of an electrical probe relative to the specimen. At intervals of $150 \mu \mathrm{m}$ the probe determined the elevation of the replica as it traversed across it, retracting $50 \mu \mathrm{m}$ from the replica surface between each measurement

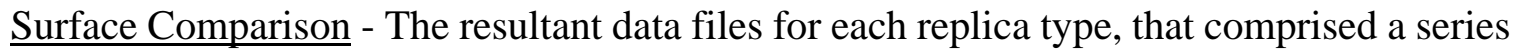
of Digital Terrain Models (DTM's) consisting of many x, y and z-co-ordinates for each 
replica were then compared using a SMADDA (Surface matching and difference detection algorithm). This comparison was carried out within a series with the object of calculating the proportion of the surface undergoing change. To this end the surface matches undertaken were:

(A) both inlays in versus both inlays out

(B) both inlays in versus large inlay out and small inlay in

(C) both inlays in versus small inlay out and large inlay in.

The proportion of the surface undergoing change was then either derived directly, from the result of match $\mathrm{A}$, or indirectly as the sums of matches $\mathrm{B}$ and $\mathrm{C}$.

A One Way Analysis of Variance was undertaken to compare the indirect results with those obtained directly.

The method of surface comparison utilised a least squares approach to surface matching in which the surfaces being compared were moved mathematically so that the surface of one DTM was superimposed upon that of another for comparative purposes. The degree of simulated tooth surface loss was then expressed as the mean overall percentage of the surface to undergo change. No change would be signified by $0 \%$ and change affecting the entire tooth surface would be represented by a figure of $100 \%$.

Full accounts of the mathematics involved in this procedure are given in Mitchell and Chadwick; 1998 and 1999.

\section{Results}


Table I details the degree of tooth surface loss found by both the direct and indirect method for each of the four replica sets. The mean percentage of the surface undergoing change as measured directly was $26.6($ S.D. $=0.6)$ compared to $26.1($ S.D. $=0.5)$ derived indirectly. A One Way Analysis of Variance of this data revealed no statistically significant $(\mathrm{P}>0.05)$ difference between the results obtained directly compared to those derived using the indirect method.

\section{Discussion}

This investigation sought to assess further the reproducibility of a process devised for the purpose of monitoring tooth surface loss. This system, as previously reported (Chadwick et al., 1997), comprised the total process chain of impression taking, replica production, surface mapping and application of a surface matching and difference detection algorithm. As each of these stages has the potential to introduce sources of error, that may adversely influence reproducibility, the authors considered it important to investigate the effects of making, mapping and comparing repeated series of the same object. Although previous work had established that the replication technique both recorded linear dimensions to within $3 \mu \mathrm{m}$ and appeared to reproduce surface topography with a constancy of $99.6 \%$ (Chadwick et al., 2002) the impact of the total process chain upon the reproducibility of a wear assessment was not made. It should be pointed out that the scenario in which the replication technique is used is to monitor the progression, or otherwise, of tooth surface loss from one epoch to another. It is therefore considered by some of no great importance if the replication process suffers small dimensional change providing this is reproducible from one sample to another, since it is a difference in contour rather than absolute values that are of interest (Williams et al., 1983). It is therefore extremely pleasing to note the 
considerable agreement (Table I) in the proportion of surface undergoing change as determined either directly or indirectly across four series of replicas involving a total of 16 replication and mapping procedures with 12 SMADDA comparisons. This represents a severe test of reproducibility.

Although the literature contains a plethora of reports upon the dimensional accuracy and reproducibility of impressions much of this information has been derived by comparing the dimensions of a standard test object to replicas cast from the impressions themselves (Bloem et al., 1991; Price et al., 1991; Boulton et al., 1996 and Nissan et al., 2000). The work reported in the present study, by both assessing topographical change directly and by derivation from a series of intermediate stages, offers an alternative approach that in the context of the scenario employed here appears to work well and evaluates all aspects of the topographical assessment process. The manufacture of a test tooth, with saucerised cavities restored with removable inlays, enabled a variety of degrees of tooth surface loss to be simulated repeatedly in a reproducible manner by the insertion/removal of these restorations in various permutations. This facilitated repeated recordings of impressions, in all states, so that a meaningful assessment of the reproducibility of the whole process could be undertaken. As this was a laboratory investigation, conducted on a prepared test tooth, the material of either the tooth or inlay is of no consequence to the investigation providing it is compatible, as in this case, with the materials of the impression and replication procedures. 
We therefore conclude that simulating wear in this way offers a more stringent test of reproducibility than comparing the dimensions of a standard test object to replicas determining tooth surface loss (wear) by the method described demonstrates high reproducibility.

\section{References}

Bloem T.J., Czerniawski B., Luke J. and Lang B.R. (1991) Determination of the accuracy of three die systems. Journal of Prosthetic Dentistry 65: 758-762.

Boulton J.L., Gage J.P., Vincent P.F. and Basford K.E. (1996) A laboratory study of dimensional changes for three elastomeric impression materials using custom and stock trays. Australian Dental Journal 41: 398-404.

Chadwick R.G., Mitchell H.L., Cameron I., Hunter B. and Tully M. (1997) Development of a novel system for assessing tooth and restoration wear. Journal of Dentistry 25: 41-47.

Chadwick R.G., Mitchell H.L. and Ward S. (2002) Evaluating the accuracy and reproducibility of a replication technique for the manufacture of electroconductive replicas for use in quantitative clinical dental wear studies. Journal of Oral Rehabilitation 29:540-545.

International Standards Organisation (1992) ISO 4823 Dental Elastomeric Impression Materials. International Standards Organisation, Geneva, Switzerland. 
Mitchell H.L. and Chadwick R.G. (1998) Mathematical shape matching as a tool in toothwear assessment - Development and Conduct. Journal of Oral Rehabilitation 25: 921-928.

Mitchell H.L. and Chadwick R.G. (1999) Digital photogrammetric concepts applied to surface deformation studies. Geomatica 53: 405-414.

National Diet and Nutrition Survey (2000) Young people aged 4 to 18 years; Vol 2; report of the oral health survey. TSO, London.

Nissan J., Laufer B-Z., Brosh T. and Assif D. (2000) Accuracy of three polyvinyl siloxane putty-wash impression techniques. Journal of Prosthetic Dentistry $\underline{83}$ : 161-165.

O’Brian M. (1994) Children’s Dental Health in the United Kingdom 1993. Office of Population Censuses and Surveys. Her Majesty’s Stationary Office, London.

Price R.B., Gerrow J.D., Sutow E.J. and MacSween R. (1991) The dimensional accuracy of 12 impression material and die stone combinations. International Journal of Prosthodontics $\underline{4}:$ 169-174. 
Williams D.F., Cunningham J., Lalor M.J., Groves D. and Atkinson J.T. (1983) Laser

techniques for the evaluation of wear in Class II restorations. Journal of Oral Rehabilitation 10:407-414. 


\section{Acknowledgement}

The work reported in this paper was funded by the Chief Scientist Office of the Scottish Executive (Grant Number K/MRS/50/C2734). The views expressed are those of the authors. 
Table 1: The proportion of the palatal surface exhibiting wear as determined directly or derived indirectly for the four sets of replicas. Wear is expressed as the mean overall percentage of the surface to undergo change.

\begin{tabular}{|c|c|c|c|c|}
\hline \multirow[t]{2}{*}{ Replica Set } & \multirow{2}{*}{$\begin{array}{l}\text { Percentage of } \\
\text { surface } \\
\text { undergoing } \\
\text { change as } \\
\text { measured } \\
\text { directly (Both } \\
\text { inlays in versus } \\
\text { Both Inlays } \\
\text { Out) }\end{array}$} & \multicolumn{3}{|c|}{$\begin{array}{l}\text { Percentage of Surface undergoing change as derived } \\
\text { from the product of the surface matches of all inlays } \\
\text { in versus (A) only large inlay out and (B) only small } \\
\text { inlay out. }\end{array}$} \\
\hline & & State A & State B & $\begin{array}{l}\text { Sum of } \\
\text { A }+B\end{array}$ \\
\hline 1 & 26.8 & 16.5 & 9.6 & 26.1 \\
\hline 2 & 25.9 & 15.4 & 10.2 & 25.6 \\
\hline 3 & 26.3 & 16.1 & 10.7 & 26.8 \\
\hline 4 & 27.5 & 15.7 & 10.0 & 25.7 \\
\hline $\begin{array}{l}\text { Mean \% Surface } \\
\text { Change } \\
\text { (S.D.) }\end{array}$ & $\begin{array}{l}26.6 \\
(0.6)\end{array}$ & & & $\begin{array}{l}26.1 \\
(0.5)\end{array}$ \\
\hline
\end{tabular}

Those values in the table body in bold are the direct and indirect values compared using

One Way Analysis of Variance. R .G. Chadwick \& S. WardThe Dental School, Park Place, Dundee, DD1 4HN, UK. 


\section{$\underline{\text { H.L. Mitchell }}$}

School of Engineering, University of Newcastle, University Drive, Callaghan, NSW 2308,

Australia. 


\section{Figure Legends}

Figure 1: Diagrammatic representation (not to scale) of the palatal aspect of the test tooth showing the location of the removable flush fitting inlays. 